Research Article

\title{
Research on Slope Deformation Prediction Based on Fractional-Order Calculus Gray Model
}

\author{
Li Li $\left(\mathbb{D},{ }^{1}\right.$ Yue Qiang $\mathbb{D},{ }^{2,3}$ Shaohong Li, ${ }^{4}$ and Zhongchao Yang ${ }^{5}$ \\ ${ }^{1}$ Civil Engineering College, Chongqing Three Gorges University, Chongqing 404100, China \\ ${ }^{2}$ Jiangxi Engineering Research Center of Water Engineering Safety and Resources Efficient Utilization, Nanchang 330099, China \\ ${ }^{3}$ Changjiang River Scientific Research Institute of Changjiang Water Resources Commission, Wuhan 430010, China \\ ${ }^{4}$ College of Environment and Civil Engineering, Chengdu University of Technology, Chengdu 610059, China \\ ${ }^{5}$ School of River and Ocean Engineering, Chongqing Jiao-tong University, Chongqing 400074, China \\ Correspondence should be addressed to Li Li; lily6636694@163.com
}

Received 11 June 2018; Revised 18 August 2018; Accepted 4 September 2018; Published 14 October 2018

Guest Editor: Jian Sun

Copyright (c) $2018 \mathrm{Li} \mathrm{Li}$ et al. This is an open access article distributed under the Creative Commons Attribution License, which permits unrestricted use, distribution, and reproduction in any medium, provided the original work is properly cited.

\begin{abstract}
Slope deformation prediction has important significance for slope prevention and control. Based on historical time series, the trend of displacement variation can be predicted in advance, and according to the development trend, risk warnings and treatment measures are proposed. The use of the mathematical model to predict slope deformation has been proved to be feasible by many studies; therefore, the choice of the predictive model and the practicability of the model are crucial issues in the prediction of slope deformation, and the mathematical prediction model used should be less complicated considering the practicality of the model. In view of slope deformation prediction, a fractional-order calculus gray model based on the coupling of gray theory and the fractional derivative method is proposed, which takes a deep foundation pit slope in Chongqing, Southwest China, as the study object. The fractional-order gray model is compared with the traditional gray models; therefore, the results show that the accuracy of slope deformation prediction based on the gray coupling model of cumulative displacement and fractional calculus is significantly higher than that of the conventional gray model, and its error is in the acceptable range compared with the actual monitoring data, which can meet the needs of engineering application. Compared with the traditional gray theory method, the gray coupling model of fractional-order calculus only increases the fractional derivative order, which is verified to be feasible, and can be used as a reference method for slope deformation prediction. It has a certain theoretical basis and a good application prospect in slope deformation prediction.
\end{abstract}

\section{Introduction}

Slope disaster prediction and forecasting is the frontier problem and research focus of current environmental geology research and is also one of the critical aspects for people to understand the natural environment $[1,2]$, with regard to geological balance of the surface, which has wide practical significance and important theoretical value. Deformation is the macroscopic and visible physical information in the process of slope evolution, and predicting slope deformation can better understand the evolution behavior of slope. Therefore, scholars have done a lot of research on slope deformation prediction and model. Generally speaking, based on the existing deformation monitoring data, nonlinear prediction is often discussed from the perspective of time series statistics or formation mechanism. Nowadays, it is more popular to apply the mathematical model for nonlinear comprehensive prediction combined with the existing data, which can further interpret the internal mechanism of slope instability, further predict the trend of deformation and failure, and provide relevant measures and treatment methods for engineering. In addition, there have been many successful cases for guiding practical projects.

At present, the slope deformation prediction is based on slope displacement monitoring data, reservoir water level change, rainfall monitoring, etc., and therefore, using certain mathematical methods or means to obtain the slope 
deformation prediction model is one of the main technical difficulties in the slope engineering. The mechanical knowledge still cannot solve the problem of slope deformation prediction properly. Therefore, mathematical methods or approaches are adopted to predict the deformation of the slope, in order to obtain the deformation trend. Foreign scholars started related research earlier. For example, Jibson [3] adopted a regression model to estimate the displacement of slope under the action of strong vibration and achieved the expected results. Kaund et al. [4] employed the backpropagation neural network model to predict slope displacement and groundwater height. At the same time, Jin et al. [5], Tan et al. [6], Zhao et al. [7], Li et al. [8], Peng et al. [9], and other domestic scholars proposed the models of slope deformation prediction in terms of slope instability and deformation mechanism, thus obtaining the optimal combination weight coefficients according to forecast effectiveness, residual error, and other parameters and carried out fitting to learn deformation prediction in order to enhance the prediction accuracy; Zhang et al. [10] and $\mathrm{Ma}$ [11] used the vector machine in mathematics to predict the slope deformation of Baima River and Ancient Tree House, which is almost the same as the actual measurement. Moreover, in respect of the prediction of landslide displacement, Huang, Cao, Zhu, Zhou, Xie, and Cai [12-17] adopted some mathematical models and intelligent methods to predict the landslides in Sichuan and Three Gorges Reservoir Area in China, which provides relevant nonengineering measures for landslide prevention and control and achieved good results. These methods used in slope or landslide deformation generally have their own advantages and disadvantages, and often different methods get different prediction results. The main differences lie in terms of the selection singleness of methods, the effectiveness of monitoring data, and the acceptability of error judgment.

Using mathematical models and intelligent evaluation methods to accurately predict slope deformation and at the same time integrating the mechanical theory interpretation with the deformation prediction has important theoretical significance and practical engineering value. The fractionalorder calculus method is an extension of the classical calculus method with memory and genetic functions, especially in the complex system description; it boasts the advantages of simple and fast modeling, easy to implementing, and accurate positioning of description and has become one of the most important tools for mathematical modeling of complex mechanical and physical processes. There are relatively few studies in this area, and among the engineering application foundations, there are a few reports on fractional creep and fractional plasticity. Wang et al. [18], Chen et al. [19], He et al. [20], and $\mathrm{Wu}$ et al. [21] came up with the fractional calculus theory to propose a nonlinear creep model for rock objects and conducted related experimental research; Tang et al. [22] put forward the creep damage model of loses with variable parameters based on fractional calculus. These studies suggest that the fractional-order calculus method can be used in geotechnical engineering research. In addition, the gray theory has the advantages of moderate sample size, quantitative qualitative combination, small computational workload, high prediction accuracy, and high accuracy. Therefore, it is a mathematical model commonly used in deformation prediction. Whether the advantages of both gray theory and fractional-order calculus can be combined and used in practical engineering prompt the necessity of research. In this paper, the fractional-order calculus method is used to derive the fractional calculus gray prediction model based on the time series of deformation data. In order to verify its rationality, taking the monitoring data of a deep foundation pit in Chongqing, Southwest China, as the engineering case example, the slope deformation prediction calculation and analysis are carried out to provide technical support for the slope deformation treatment and maintenance.

\section{Principles and Methods}

2.1. Derivation of Fractional-Order Calculus Gray Model of Slope Deformation Prediction. Assume that the slope deformation monitoring data are $X^{*}=\left(X^{*}(1), X^{*}(2), \cdots\right.$, $X^{*}(n)$ ), and use the appendix Lemma 1 and Lemma 2 to establish a fractional gray difference formula:

$$
\alpha^{(1)} X^{(1-p)}(k)+a Z^{(0)}(k)=b,
$$

where $\alpha^{(1)} X^{(1-p)}(k)(k=1,2, \cdots, n)$ denotes the $p$ order $(0 \leq p \leq 1)$ difference of $X^{(0)}(k)$; that is, $X^{(0)}(k)$ is added to $1-p$ first and then, $X^{(1-p)}(k)$ is differentiated by the first order: $\alpha^{(1)} X^{(1-p)}(k)=X^{(1-p)}(k)-X^{(1-p)}(k-1)$,

$$
Z^{(0)}(k)=\frac{X^{(0)}(k)+X^{(0)}(k+1)}{2} .
$$

Derivate parameters $a$ and $b$ according to the least squares method (see Appendix Lemma 4 for details):

$$
\begin{aligned}
{\left[\begin{array}{l}
a \\
b
\end{array}\right] } & =\left(B^{T} B\right)^{-1} B^{T} Y, \\
B & =\left[\begin{array}{cc}
-Z^{0}(2) & 1 \\
-Z^{0}(3) & 1 \\
\vdots & \vdots \\
-Z^{0}(n) & 1
\end{array}\right], \\
Y & =\left[\begin{array}{c}
\alpha^{(1)} X^{(1-p)}(2) \\
\alpha^{(1)} X^{(1-p)}(3) \\
\vdots \\
\alpha^{(1)} X^{(1-p)}(n)
\end{array}\right] .
\end{aligned}
$$

The time response of the descendant into the fractional gray model (see Appendix Lemma 5 for details) is

$$
X^{(0)}(t)=b \sum_{k=0}^{\infty} \frac{(-a)^{k} t^{k+p}}{\Gamma(k p+p+1)},
$$

where $t$ is the response time and the unit is the day. The above formula is a fractional gray model for slope deformation prediction. 


\subsection{Implementation of Fractional Calculus Gray Model for} Slope Deformation Prediction. According to the above mathematical lemma and derivation, the detailed steps of the fractional-order calculus gray model of slope deformation are as follows:

(1) First of all, process the original displacement data, and subtract the initial value from the existing time series data so that the initial value is 0 . This is to directly apply the solution formula of Lemma 5 . In this step, it should be noted that the postprocessing time series data cannot be negative because usually the cumulative displacement for slope deformation prediction is adopted, and thus, it can be satisfied.

(2) Determine the order of the fractional order, and apply the parameters of the lemma gray model.

(3) Substitute the parameters into the solution of the albinism differential equation of the gray model, adding the initial values to all the simulated values, thus obtaining the predicted value of the slope deformation.

In the above derivation, the derivative order $p$ value needs to be artificially given. In order to obtain a reasonable $p$ value, an optimization algorithm (such as the gradient method, genetic algorithm, and particle swarm algorithm) is used to solve the problem. Therefore, the solution of the $p$ value can be expressed as

$$
\begin{array}{ll}
\min & \left\|X^{(0)}(t)-X^{(\exp )}(t)\right\|_{1}, \\
\text { s.t. } & p \in[0,1],
\end{array}
$$

where $X^{(\exp )}(t)$ is the monitoring data and \|\|$_{1}$ represents first-norm.

In order to facilitate visual reading and make it easy to use, the Matlab toolbox is called to compile the corresponding program, and the implementation process is shown in Figure 1.

\section{Engineering Example of Slope Deformation Prediction of a Deep Foundation Pit}

\subsection{Survey of Engineering Geology of the Deep Foundation Pit} Slope. The deep foundation pit slope project of an international garden in Chongqing is located in the Northern New District of Chongqing. It covers an area of about $1,200 \mathrm{mu}\left(1 \mathrm{mu} \approx 666.66 \mathrm{~m}^{2}\right)$, of which the pure water surface area of the lake is about $160 \mathrm{mu}$. The terrain falls on the lakeside like a gentle slope, wherein Region B covers $300 \mathrm{mu}$, which is located in the central lakeside hinterland of the project.

This region belongs to the subtropical monsoon climate area, its annual average rainfall is $1100 \mathrm{~mm}$, and the rainfall is mostly concentrated from May to September, taking up $70 \%$ of the annual rainfall; the long-time average annual temperature is $18.6^{\circ} \mathrm{C}$, the extreme minimum temperature is $4.5^{\circ} \mathrm{C}$, and the extreme maximum temperature is $42.5^{\circ} \mathrm{C}$; the long-time average annual relative humidity is $80 \%$, and the annual distribution is the largest in December, namely, $87 \%$, and the smallest in August, namely, 74\%; and the frost

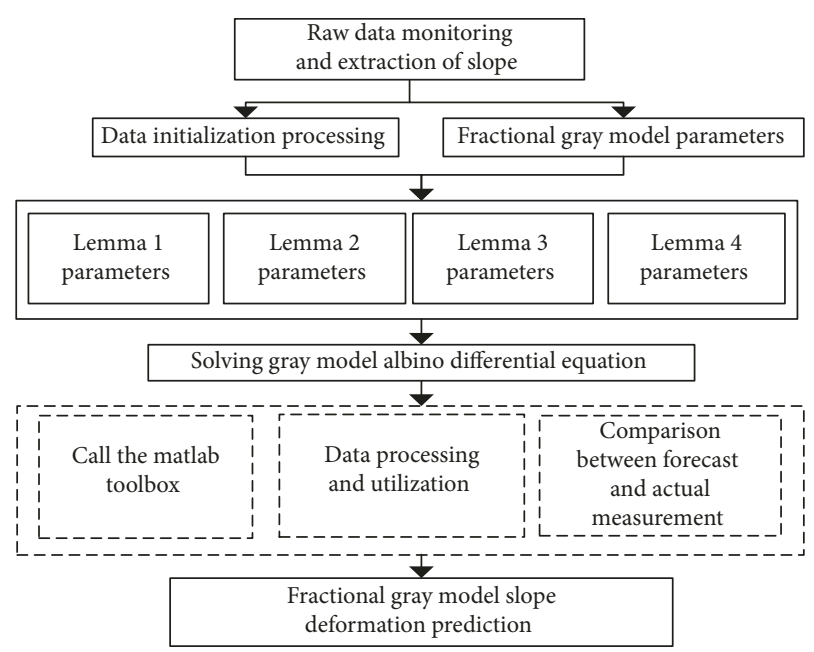

FIgURE 1: Fractional-order calculus gray model implementation process.

period generally lasts 10 20 days, and fog days are up to 20 35 days, and the number of sunshine hours is as many as $1384.2 \sim 1542.8$ hours.

The area is a type of denuded hilly landform, with no faults passing through it. The occurrence of rock formation is SW $310^{\circ} \angle 9^{\circ}$ in the exposed area of the bedrock near the area, and there are two sets of fissures in the rock mass. The structural fissures are not developed, with no faults passing through it, and the development degree of fissures is simple. The slope angle of the terrain is $2 \sim 28^{\circ}$; the average slope angle is about $22^{\circ}$; the topography and geomorphology are relatively complex; the dip angle of the rock layer is gentle, with no faults passing through it; the fissures are not developed; the rock mass takes on the shape of the medium thick to thick layer; the lithology and soil layer combination are binary combination, and the exposed bedrocks are the mud rocks and sand stones of Middle Jurassic Upper Shaximiao Formation (J2s); the quaternary soil layer includes el-dlQ (Q4el + dl), Qml (Q4ml), and Qapl (Q4al + pl); the soil layer is 0.50 to $4.7 \mathrm{~m}$ thick $(2.7 \mathrm{~m}$ on average), and the dip angle of the geosynthetic interface is $3 \sim 14$ degrees; and the local geotechnical interface is forward and nonemptive, and dip size is $\geq 20^{\circ}$, accounting for $28.8 \%$ of the planning area. The relationship between the rock mass penetrating the structural plane and the oblique (edge) is relatively complicated, and the influence of surface water and groundwater on the rock mass is small, the basic intensity of earthquakes of the VI degree. Human activities that affect the geological environment are not strong. There are no bad geological phenomena such as dangerous rock collapse, landslide, debris flow, and ground collapse, and thus, the geological environment is complex to some degree.

According to the engineering geological conditions, three kinds of protection methods are used to support and protect the slope of the deep foundation pit, such as protection treatment of the lattice anchor rod or ordinary slideresistant pile or slide-resistant pile with prestressed anchor cable by combining with the C25 shotcrete panel. In the process of construction, monitor the horizontal and vertical 
displacements of the slope top, monitor the anchor rod and prestressed anchor cable press, and make a macroscopic inspection of the Earth's surface.

\subsection{Acquisition and Analysis of Deformation Monitoring} Data. The safety grade of the foundation pit slope in Region B of an international garden in Chongqing is Grade I, the whole excavation panorama of the deep foundation pit slope is shown in Figure 2(a), and the monitoring plane graph is shown in Figures 2(b) and 2(c). During the construction process, before the foundation pit is excavated to $5 \mathrm{~m}$, monitor at the frequency of once every 7 days; before the whole foundation pit is excavated from $5 \mathrm{~m}$ to $10 \mathrm{~m}$, monitor at the frequency of once every 3 days; and when the excavation of the whole foundation pit slope exceeds $10 \mathrm{~m}$, monitor at the frequency of once every day. The horizontal displacement and the displacement of the pile top are tested with a total station instrument by means of the photoelectrode coordinate method, and the datum point needed to monitor the deformation observation point accords with second-order accuracy. At the time of settlement observation, the error in the observation station's height difference of the observation point is $\leq 0.5 \mathrm{~mm}$ and in the observation of plane displacement, the error in the coordinates of observation points is $\leq 3 \mathrm{~mm}$. Two observation points are set on Building No. Six, the Third Phase of the southern slope of the foundation pit in this region, and they are named as JC1 and JC2. The measured deformation values of the JC1 and JC2 monitoring points are all $5 \mathrm{~m}$ in foundation pit depth, and the monitoring time is concentrated from June to September, during which the seasonal rainfall is relatively obvious. Therefore, the change in the range of the reservoir water level in the central lakeside hinterland is relatively large, and the actual monitoring data are shown in Figure 3.

3.3. Analysis of Deformation Prediction Results. Select the first 50 time series data of the JC-1 monitoring point to build the model, and predict the displacement changes of the following 26 time series; and select the first 30 time series data of the JC-2 monitoring point to build the model, and predict the displacement changes of the following 15 time series. Among them, the JC-1 monitoring point is located at the top of the deep foundation pit, and the deep foundation pit of the JC- 2 monitoring point is located at the bottom position. The sudden change of the monitoring point JC-1 at $10 \sim 20$ days is mainly due to the local temporary loading effect at the top of the slope.

According to the measured data of JC-1 and JC-2 monitoring points, using the fractional calculus gray prediction model and gray prediction model, the $p$ value was obtained by the genetic algorithm in the calculation, the $p$ value of JC- 1 was taken as 0.5 , and the $p$ value of JC-2 was taken as 0.6, and the results are shown in Figure 4. From the deformation prediction of JC-1 and JC- 2 monitoring points, the deformation prediction value calculated by the fractional-order calculus gray model is smaller than the measured value on the whole, the deformation prediction value calculated by the gray model is larger than the measured value on the whole, the overall variation trends of the deformation prediction values calculated by the fractionalorder calculus gray model and gray model are the same, and there is no apparent singularity. With the increase of the time series of the deformation monitoring point, the absolute error value of the deformation prediction of the fractional calculus gray model and the measured value are gradually increased, and the absolute error value margin of the deformation prediction of the gray model and the measured value are getting larger and larger; the gradually accumulated value is getting bigger and bigger. When calculating the fractional-order calculus gray model, the minimum absolute error of the JC-1 monitoring point is $2.02 \mathrm{~mm}$, appearing in the first half of monitoring time series prediction, the maximum absolute error of it is $3.98 \mathrm{~mm}$, appearing in the last phase of monitoring time series prediction, and the front-to-rear difference reaches $1.96 \mathrm{~mm}$; the minimum absolute error of the JC- 2 monitoring point is $0.125 \mathrm{~mm}$, appearing in the middle section of monitoring time series prediction, the maximum absolute error of it is $0.709 \mathrm{~mm}$, appearing in the last phase of monitoring time series prediction, and the front-to-rear difference reaches $0.584 \mathrm{~mm}$; the variation trends and laws of absolute errors of JC- 1 and JC-2 monitoring points are basically the same. With the increase of monitoring time series, the absolute error increases gradually, and the difference between the maximum and minimum values of the absolute error is gradually on the rise as well. When calculating the gray model, the minimum absolute error of JC-1 is $3.79 \mathrm{~mm}$, appearing at the first time series point of monitoring time series prediction, and the maximum absolute error of it is $8.58 \mathrm{~mm}$, appearing at the last time series point of monitoring time series prediction, and the front-to-rear difference reaches $4.79 \mathrm{~mm}$; the minimum absolute error of JC-2 is $0.467 \mathrm{~mm}$, appearing at the first time series point of monitoring time series prediction, the maximum absolute error of it is $1.843 \mathrm{~mm}$, appearing at the last time series point of monitoring time series prediction, and the front-to-rear difference reaches $1.376 \mathrm{~mm}$; the variation trends and laws of absolute errors of JC- 1 and JC- 2 monitoring points are basically the same. Comparing the deformation prediction of the fractional-order calculus gray model and gray model, the absolute error calculated by the gray model is monotonically increasing, while the absolute error calculated by the fractional-order calculus gray model first increases, then decreases, and finally increases. The absolute error of the gray model is one time larger than that of the fractionalorder calculus gray model. In order to make a clearer comparison, the relative errors of the measured data and the predicted calculation data of JC- 1 and JC- 2 monitoring points are calculated. As shown in Figure 5, the variation trends and laws of relative errors of JC- 1 and JC- 2 monitoring points are basically the same as that of the absolute errors which will not be elaborated here in detail.

\subsection{Discussion}

(1) Analysis of the influence of the $p$ value on prediction results. The method for determining the 


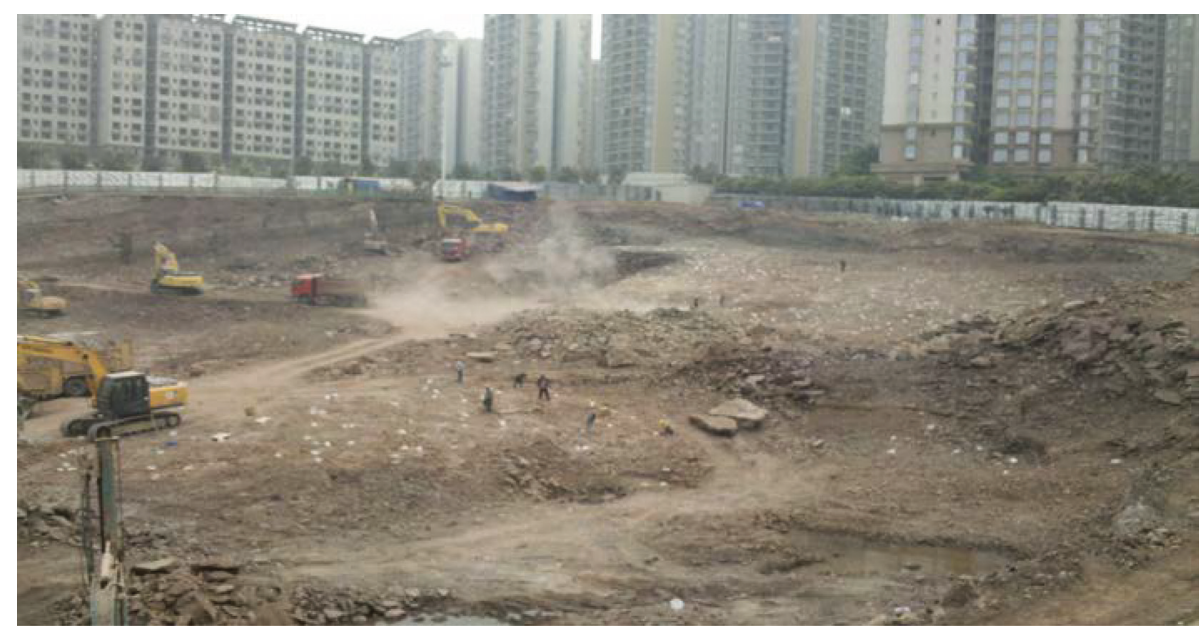

(a)

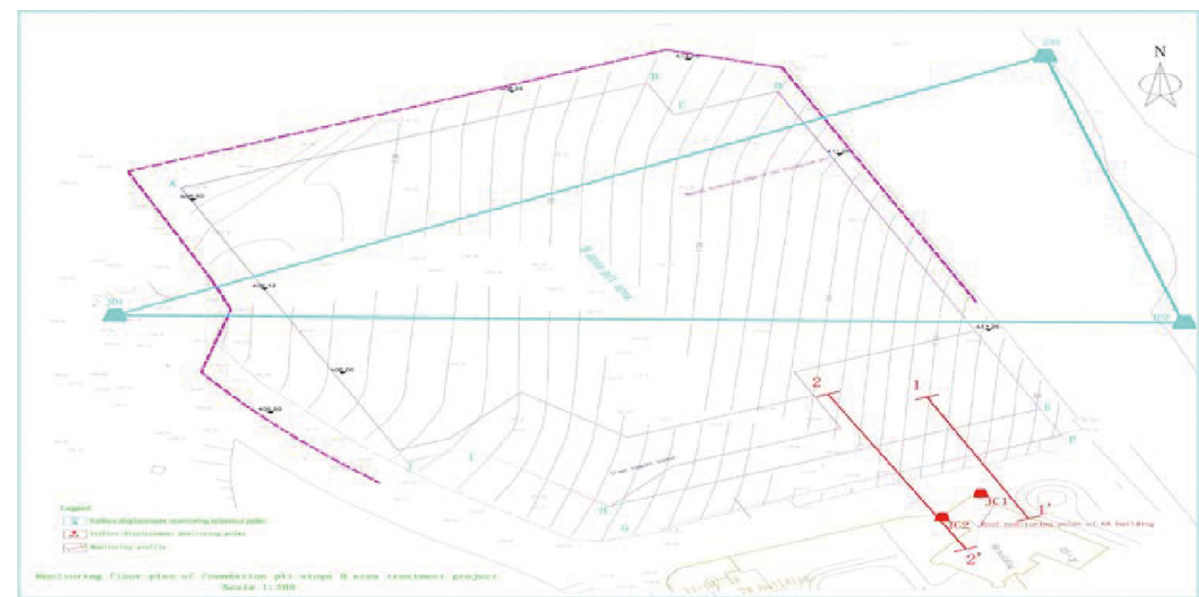

(b)

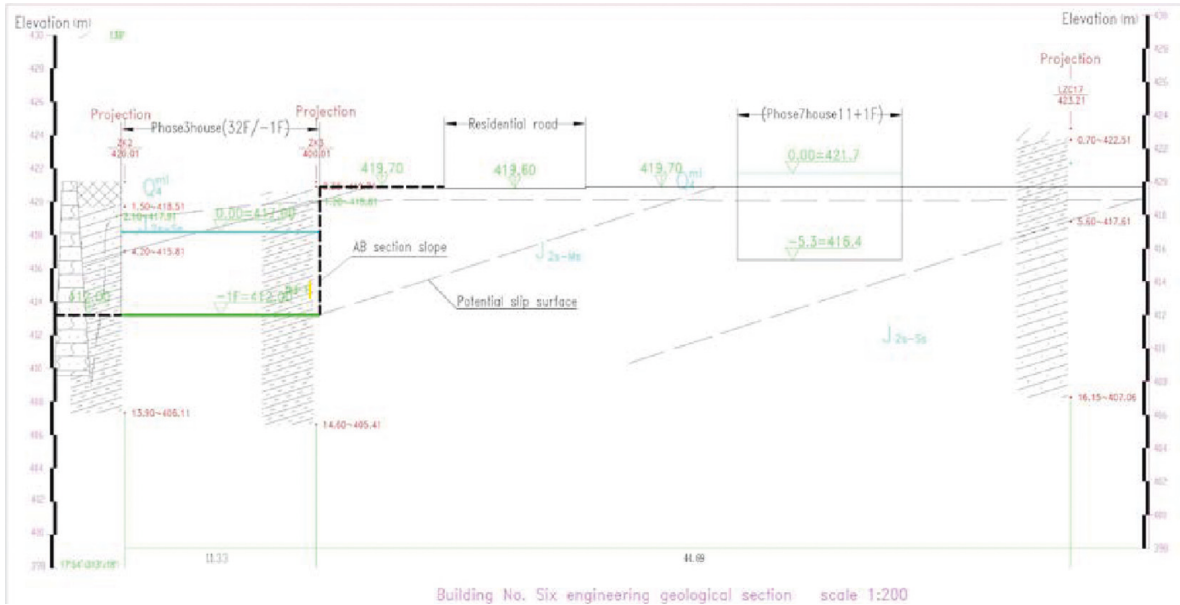

(c)

FIGURE 2: Deep foundation pit slope, monitoring layout, and engineering geological section: (a) whole panorama of deep foundation pit slope; (b) monitoring floor plan of foundation pit slope B district treatment project; (c) Building No. Six engineering geological section.

$p$ value is given above. The impact of different $p$ values on the model prediction results is further analyzed. Figure 6 shows the sensitivity analysis of different $p$ values for the settlement results of the model using JC-2 monitoring points as an example. It can be seen from Figure 6 that different $p$ values have a greater impact on the model prediction results. In general, as the $p$ value increases, the overall value of the prediction result decreases. 


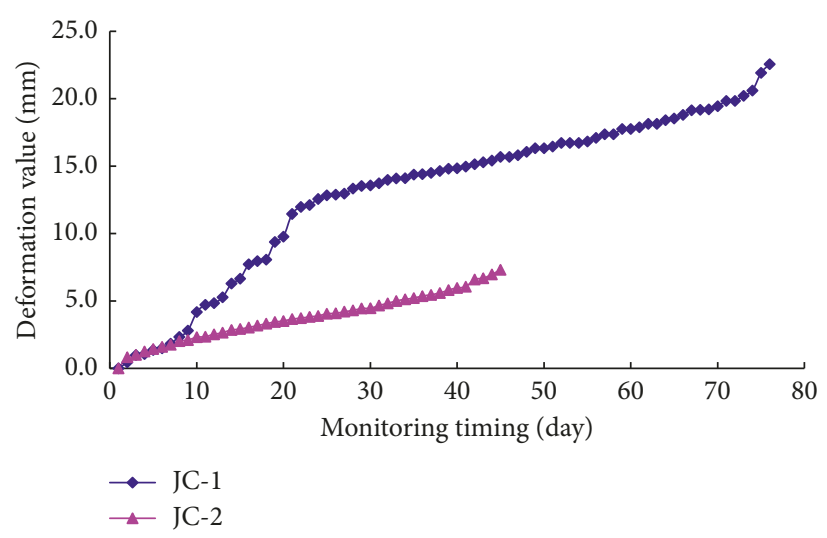

FIGURE 3: Actual measurement data of JC-1 and JC-2 monitoring points of a deep foundation pit slope.
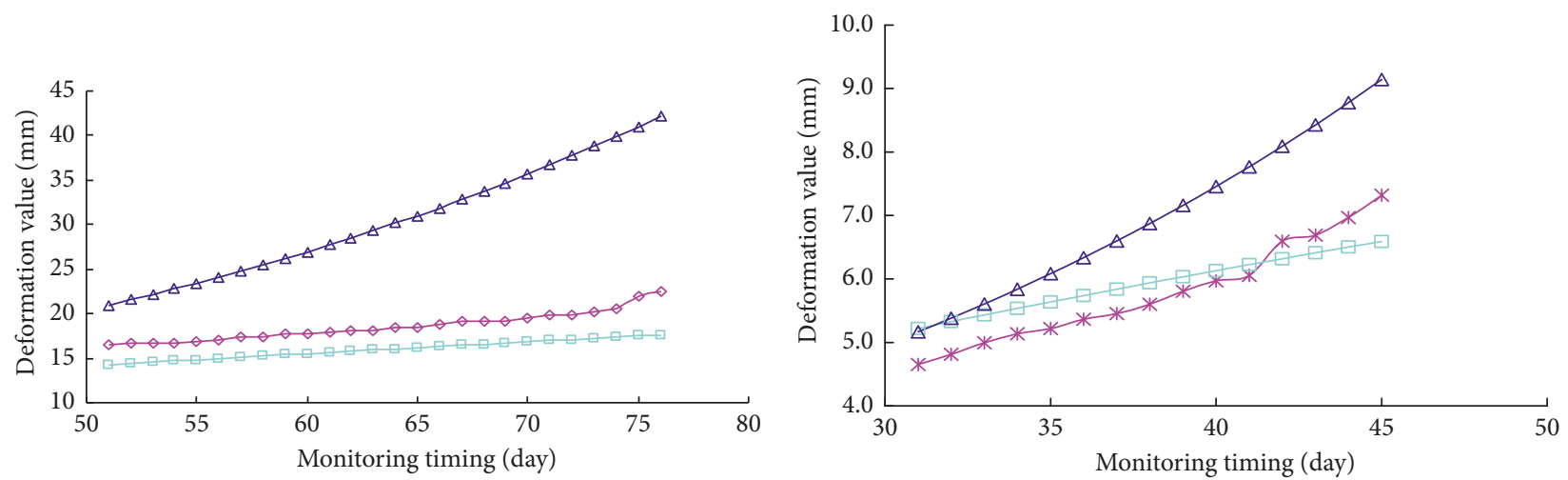

$\multimap$ Measured value

$\because$ Fractional-order calculus gray model prediction

$\triangle$ Gray model prediction

(a)

* Measured value

$\square$ Fractional-order calculus gray model prediction

$\triangle$ Gray model prediction

(b)

FIgURE 4: (a) JC-1 and (b) JC-2 monitoring point deformation predictions.

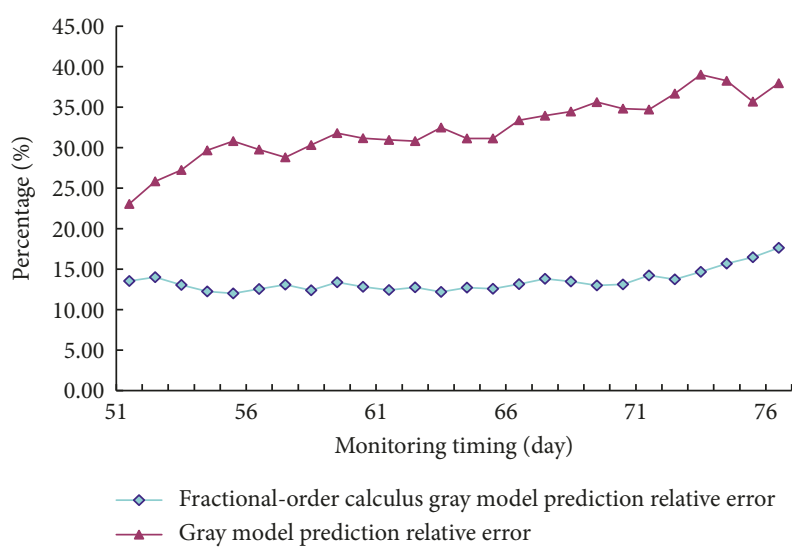

(a)

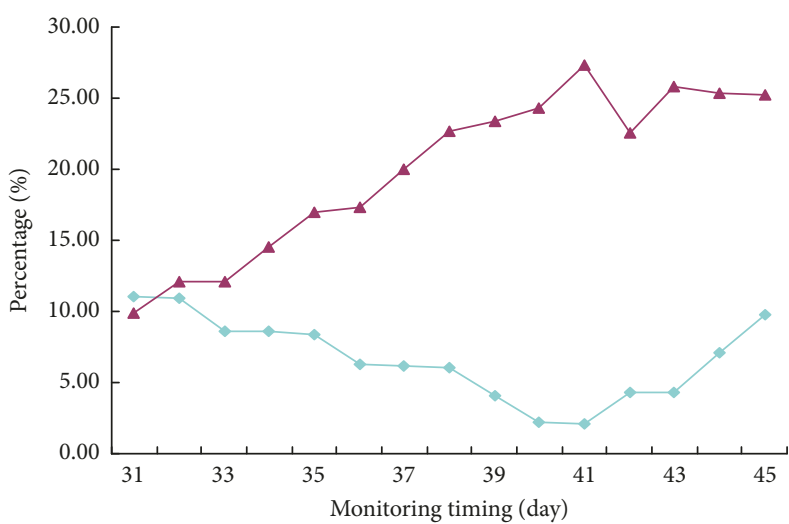

$\ldots$ Fractional-order calculus gray model prediction relative error —- Gray model prediction relative error

(b)

Figure 5: (a) JC-1 and (b) JC-2 monitoring point deformation prediction relative errors. 


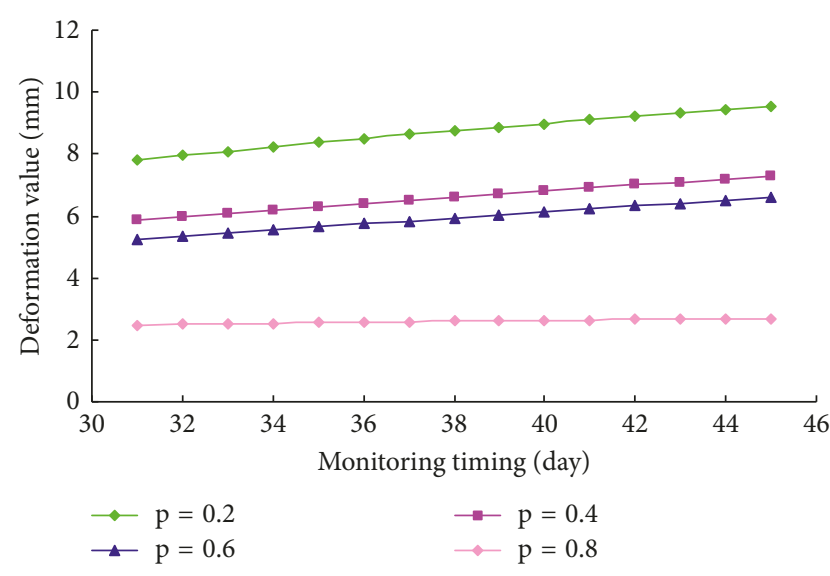

Figure 6: Parameter $p$ sensitivity analyses of the JC-2 monitoring point.

(2) Different from the traditional gray model, the fractional-order gray model introduces the derivative order $p$, which makes the traditional gray model be a special case of the fractional-order gray model. Therefore, the fractional-order gray model can better fit the original sequence with strong nonlinearity. From the calculation example in this paper, the prediction accuracy of the fractional-order gray model is obviously improved compared with the existed gray model, but it still has a certain gap from the real monitoring value. In addition, the determination of the $p$ value requires the aid of an optimization algorithm, which requires more calculation amount of the model than that of the traditional gray model.

\section{Conclusions}

(1) Slope deformation is closely related to soil properties and engineering geological environment. Rainwater erosion and slope load may directly accelerate slope deformation. Therefore, the analysis of the development trend of slope deformation needs to consider the internal and external factors. It is often combined with the existing monitoring data to use mathematical models to carry out nonlinear calculations and predict the trend of change, which can provide technical support for slope management and promote the disaster prevention and reduction.

(2) With the fractional-order calculus method, based on the deformation monitoring data time series, from the theoretical derivation point of view, the fractional-order calculus gray model is proposed after analysis and derivation, and the applicable conditions and ranges are nonnegative sequences, and the initial value is 0 . The steps of using the fractional-order calculus gray prediction model are given. Using the Matlab language to compile the corresponding program, these corresponding functions can be realized one by one.
(3) Based on the established fractional-order calculus gray model method, the monitoring data of a deep foundation pit slope in Chongqing, Southwest China, was selected as an example to verify the model. The calculation results show that the fractional-order calculus method has better prediction effect than that of the gray model, and the latter has a maximum relative error of $37.96 \%$, while the former has a maximum relative error of $17.63 \%$. These results show that the fractional-order gray model has a certain application prospect in the prediction of mountain slope deformation. Compared with the measured data, the relative errors are within the acceptable range, which can provide reference for similar projects.

\section{Appendix}

Lemma 1. Assume that the nonnegative sequence is $X^{*}$, and $X^{r}(k)=\sum_{i=1}^{k} X^{r-1}(i)$ is a r-order additive operator, then, $C_{r-1}^{k}=1, C_{k}^{k+1}=0$, it is $X^{r}(k)=\sum_{i=1}^{k} C_{k-i+r-1}^{k-i} X^{0}(i)$. The lemma can be proved by mathematical induction, omitted here.

Lemma 2. In Lemma 1, when $r$ is a fraction, the result is unchanged, and the coefficient becomes

$$
C_{k-i+r-1}^{k-i}=\frac{[k-i+r-1][k-i+r-2] \cdots[r+1] r}{(k-i) !}
$$

Lemma 3. The fractional calculus gray model is

$$
\alpha^{(1)} X^{(1-p)}(k)+a Z^{(0)}(k)=b .
$$

In the formula, $\alpha^{(1)} X^{(1-p)}(k)$ represents the corresponding p-order $(0<p<1)$ difference of $X^{(0)}(k)$. First, $X^{(0)}(k)$ is accumulated in $1-p$ order, and the first-order difference equation is used for $X^{(1-p)}(k)$. Hence, $\alpha^{(1)} X^{(1-p)}(k)=X^{(1-p)}(k)-$ $X^{(1-p)}(k-1) ; Z^{(0)}(k)=X^{(0)}(k)+X^{(0)}(k+1) / 2$.

Lemma 4. The Least Squares estimate of Lemma 3 satisfies

$$
\begin{aligned}
{\left[\begin{array}{l}
a \\
b
\end{array}\right] } & =\left(B^{T} B\right)^{-1} B^{T} Y, \\
B & =\left[\begin{array}{cc}
-Z^{0}(2) & 1 \\
-Z^{0}(3) & 1 \\
\vdots & \vdots \\
-Z^{0}(n) & 1
\end{array}\right], \\
Y & =\left[\begin{array}{c}
\alpha^{(1)} X^{(1-p)}(2) \\
\alpha^{(1)} X^{(1-p)}(3) \\
\vdots \\
\alpha^{(1)} X^{(1-p)}(n)
\end{array}\right] .
\end{aligned}
$$


Lemma 5. The solution of the fractional partial differential equation $d^{p} X^{(0)}(t) / d t+a X^{(0)}(t)=b$ solution is

$$
X^{(0)}(t)=b \sum_{k=0}^{\infty} \frac{(-a)^{k} t^{k+p}}{\Gamma(k p+p+1)} .
$$

The equation is Laplace positive transformation:

$$
S^{p} E(S)=+a E(S)=\frac{b}{S} \text {. }
$$

So,

$$
E(S)=\frac{b}{S(S p+\alpha)} .
$$

Laplace transformation by $M-L$ function is

$$
L\left\{t^{\alpha k_{1}+\beta-1} E_{\alpha, \beta}^{(k)}\left( \pm \alpha t^{\alpha}\right) ; s\right\}=\frac{k_{1} ! S^{\alpha-\beta}}{\left(S^{\alpha} \mp \alpha\right)^{k_{1}+1}} .
$$

Comparing Formula (10a) and Formula (11), and so, $k=0, \alpha=p, \beta=p+1$.

The inverse of Equation (10b) is in the form

$$
\begin{aligned}
X^{0}(t) & =b t^{p} \sum_{k=0}^{\infty} \frac{\left(-a t^{p}\right)^{k}}{\Gamma(p+1+k p)} \\
& =b \sum_{k=0}^{\infty} \frac{(-a)^{k} t^{p k+p}}{\Gamma(p+1+k p)} \\
& =b \sum_{k=0}^{\infty} \frac{(-a)^{k} t^{p k+p}}{\Gamma(p(k+1)) p(k+1)} .
\end{aligned}
$$

Proof completed.

\section{Data Availability}

The data used to support the findings of this study are available from the corresponding author upon request.

\section{Conflicts of Interest}

The authors declare that they have no conflicts of interest.

\section{Authors' Contributions}

Li Li and Yue Qiang are the first authors.

\section{Acknowledgments}

The study is performed within the Changjiang River Scientific Research Institute of Changjiang Water Resources Commission Open Research Program (program no. ckwv2016393/KY), the Open Foundation of Jiangxi Engineering Research Center of Water Engineering Safety and Resources Efficient Utilization (Grant no. OF201605), Three Gorges Reservoir Ecological Environment Protection and Disaster Prevention (2011 collaborative innovation center of Chongqing), Scientific and Technological Research Program of Chongqing Municipal Education Commission (Grant nos. CXTDX201601034 and
KJQN201801224), Science and Technology Talent Special Fund Project of Wan-Zhou District, Chongqing (Grant no. 2016027), Three Gorges Reservoir Area Project Structure Disaster Prevention and Reduction and Safety Research Innovation Team of Chongqing Three Gorges University, Outstanding Scientific and Technological Achievements into Cultivation Project (Grant no. 17zh01), and Field Grade Major Breeding Program of Chongqing Three Gorges University (Grant no. 15ZP04)

\section{References}

[1] W. Du and G. Wang, "One-step newmark displacement model for probabilistic seismic slope displacement hazard analysis," Engineering Geology, vol. 205, pp. 12-23, 2016.

[2] W. Du and G. Wang, "Fully probabilistic seismic displacement analysis of spatially distributed slopes using spatially correlated vector intensity measures," Earthquake Engineering and Structural Dynamics, vol. 43, no. 5, pp. 661-679, 2014.

[3] R. W. Jibson, "Regression models for estimating coseismic landslide displacement," Engineering Geology, vol. 91, no. 2-4, pp. 209-218, 2007.

[4] R. B. Kaunda, R. B. Chase, A. E. Kehew, K. Kaugars, and J. P. Selegean, "Neural network modeling applications in active slope stability problems," Environmental Earth Sciences, vol. 60, no. 7, pp. 1545-1558, 2010.

[5] H. Y. Jin and W. Y. Xu, "Prediction of slope deformation modes with weighted function combination method," Journal of Engineering Geology, vol. 16, no. 4, pp. 518-521, 2008.

[6] X. L. Tan, W. Y. Xu, D. W. Liu, and D. W. Cai, "Hybrid prediction model for high slope displacement and its application," Journal of Hydraulic Engineering, vol. 41, no. 3, pp. 294-299, 2010.

[7] M. H. Zhao, J. H. Liu, B. C. Chen, and D. Q. Liu, "Variableweight combination forecasting model of slope deformation and instability," Rock and Soil Mechanics, vol. 28, pp. 553-557, 2007.

[8] C. Li, Q. H. Jiang, C. B. Zhou, and Z. F. Qi, "Forecasting model of slope stability considering deformation mechanism," Rock and Soil Mechanics, vol. 32, pp. 545-550, 2011.

[9] L. Peng, R. Q. Niu, and T. Wu, "Time series analysis and support vector machine for landslide displacement prediction," Journal of Zhejiang University Engineering Science, vol. 47, no. 9, pp. 1672-1679, 2013.

[10] J. Zhang, K. L. Yin, J. J. Wang, and F. M. Huang, "Displacement prediction of Baishuihe landslide based on time series and PSO-SVR model," Journal of Rock Mechanics and Engineering, vol. 34, no. 2, pp. 382-391, 2015.

[11] W. T. Ma, "Forecasting slope displacements based on gray least square support vector machines," Rock and Soil Mechanics, vol. 31, no. 5, pp. 1670-1674, 2010.

[12] Y. Cao, K. L. Yin, D. E. Alexander, and C. Zhou, "Using an extreme learning machine to predict the displacement of steplike landslides in relation to controlling factors," Landslides, vol. 13, no. 4, pp. 725-736, 2016.

[13] F. M. Huang, J. S. Huang, S. H. Jiang, and C. B. Zhou, "Landslide displacement prediction based on multivariate chaotic model and extreme learning machine," Engineering Geology, vol. 218, pp. 173-186, 2017.

[14] X. Zhu, Q. Xu, M. G. Tang, W. Nie, S. Q. Ma, and Z. P. Xu, "Comparison of two optimized machine learning models for predicting displacement of rainfall-induced landslide: a case study in Sichuan Province, China," Engineering Geology, vol. 218, pp. 213-222, 2017. 
[15] C. Zhou, K. L. Yin, Y. Cao, and B. Ahmed, "Application of time series analysis and PSO-SVM model in predicting the Bazimen landslide in the Three Gorges Reservoir, China," Engineering Geology, vol. 204, pp. 108-120, 2016.

[16] S. F. Xie, Y. J. Liang, Z. T. Zheng, and H. F. Liu, "Combined forecasting method of landslide deformation based on MEEMD, approximate entropy, and WLS-SVM," International Journal of Geo-Information, vol. 6, no. 1, p. 5, 2017.

[17] Z. L. Cai, W. Y. Xu, Y. D. Meng, C. Shi, and R. B. Wang, "Prediction of landslide displacement based on GA-LSSVM with multiple factors," Bulletin of Engineering Geology and the Environment, vol. 75, no. 2, pp. 637-646, 2016.

[18] J. B. Wang, X. R. Liu, and T. H. Wang, "A nonlinear creep model for rocks based on modified fractional viscous body," Journal of Central South University (Science and Technology), vol. 46, no. 4, pp. 293-299, 2015.

[19] L. Chen, S. G. Chen, H. Zhang, and J. S. Yang, "A nonlinear viscoelasto-plastic creep model based on fractional calculus," Journal of Sichuan University (Engineering Science Edition), vol. 45, no. 3, pp. 10-14, 2013.

[20] Z. L. He, Z. D. Zhu, M. L. Zhu, and Z. J. Li, “An unsteady creep constitutive model based on fractional-order derivatives," Rock and Soil Mechanics, vol. 37, no. 3, pp. 737-775, 2016.

[21] F. Wu, H. P. Xie, J. F. Liu, Y. Bian, and J. L. Pei, "Experimental study of fractional viscoelastic-plastic creep model," Chinese Journal of Rock Mechanics and Engineering, vol. 33, no. 5, pp. 964-970, 2014.

[22] H. Tang, D. P. Wang, Z. Duan, F. S. Zhao, F. Song, and D. Qi, "Variable parameters creep damage model of loess based on fractional calculus," Journal of Central South University (Science and Technology), vol. 46, no. 11, pp. 4248-4255, 2015. 


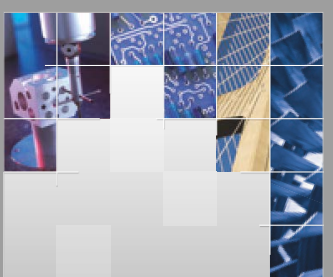

\section{Enfincering}
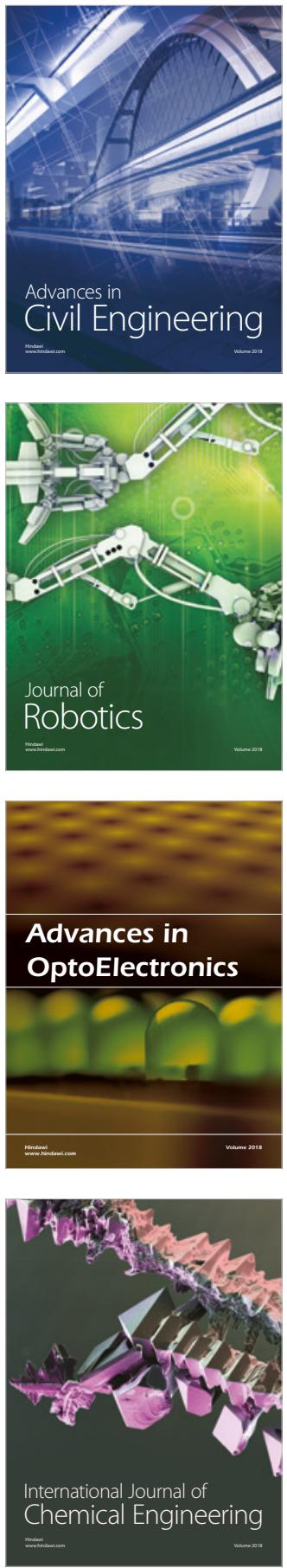

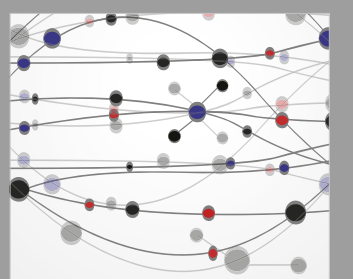

\section{Rotating \\ Machinery}

The Scientific World Journal

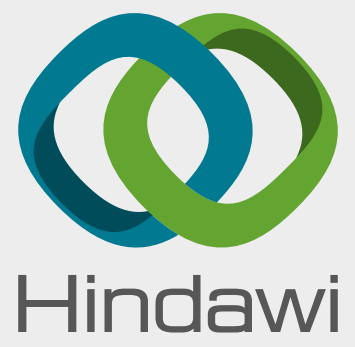

Submit your manuscripts at

www.hindawi.com
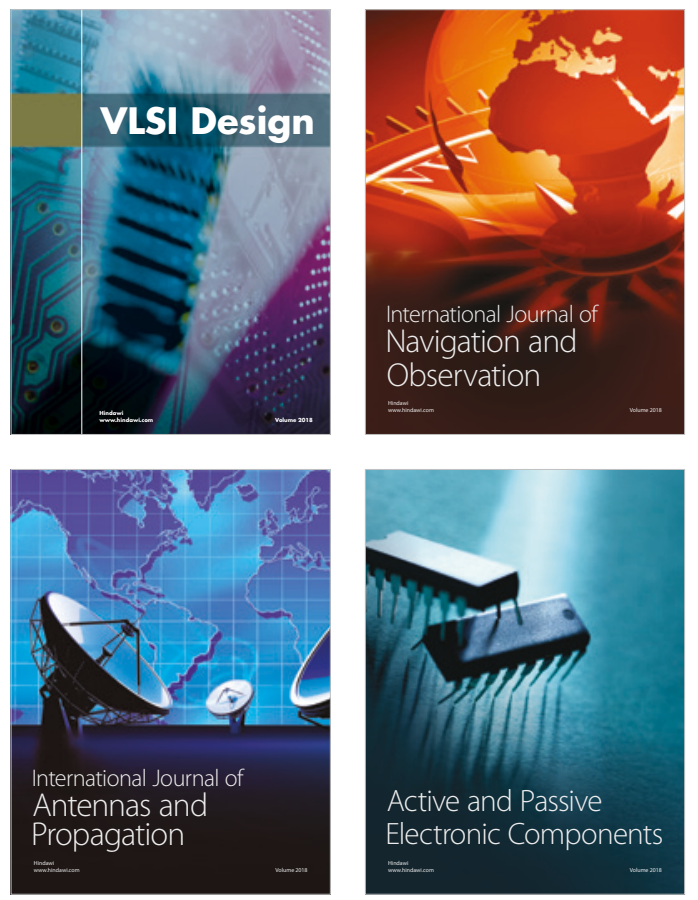
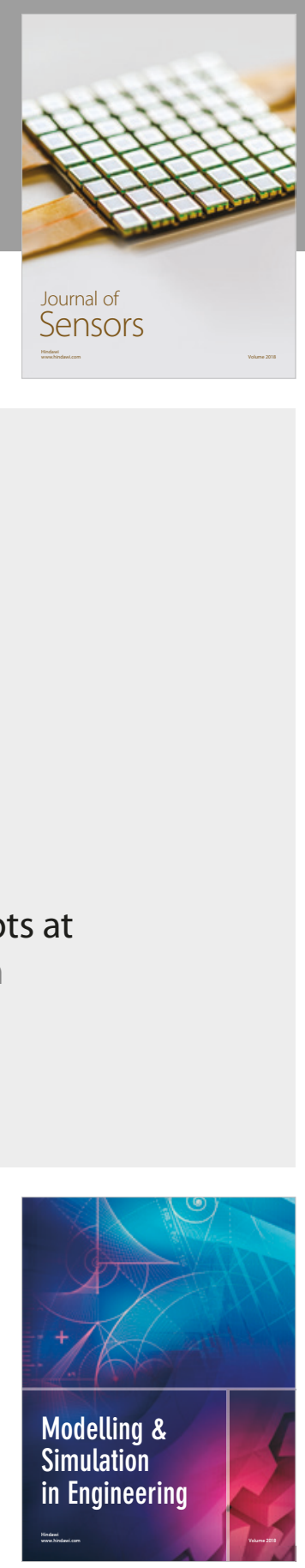

\section{Advances \\ Multimedia}
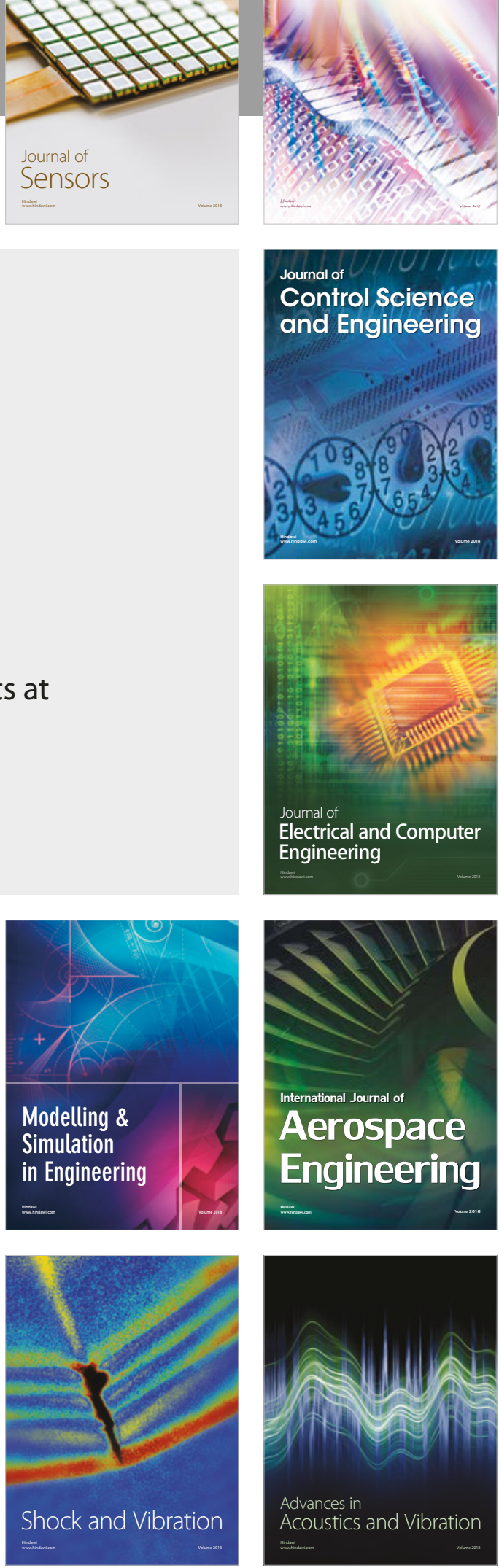